Pacific

Journal of

Mathematics

\title{
FUSION AND FISSION IN GRAPH COMPLEXES
}

\author{
James Conant
}




\title{
FUSION AND FISSION IN GRAPH COMPLEXES
}

\author{
JAMES CONANT
}

\begin{abstract}
We analyze a functor from cyclic operads to chain complexes first considered by Getzler and Kapranov and also by Markl. This functor is a generalization of the graph homology considered by Kontsevich, which was defined for the three operads Comm, Assoc, and Lie. More specifically we show that these chain complexes have a rich algebraic structure in the form of families of operations defined by fusion and fission. These operations fit together to form uncountably many Lie $_{\infty}$ and co-Lie ${ }_{\infty}$ structures. In particular, the chain complexes have a bracket and cobracket which are compatible in the Lie bialgebra sense on a certain natural subcomplex.
\end{abstract}

\section{Introduction.}

More than a decade ago Maxim Kontsevich $[\mathbf{K}]$ considered graph homology as a tool for studying and computing the homology of many seemingly disparate objects. One version of the graph complex computes, via work of R.C. Penner $[\mathbf{P}]$, the homology of the moduli space (or equivalently mapping class group) of surfaces. Another version computes, via work of M. Culler and K. Vogtmann $[\mathbf{C u V}]$, the homology of the group of outer automorphisms of the free group. There is also a version which is related to finite type invariants of three-manifolds. On the other hand, these three graph complexes compute the homology of three infinite dimensional Lie algebras, leading to quite unexpected isomorphisms. Kontsevich's graph complexes were generalized to the case of modular operads by Getzler and Kapranov[GK2], and were considered for the special case of cyclic operads by Martin Markl [M].

In $[\mathbf{C V}]$ Karen Vogtmann and I showed that the commutative graph complex carries the structure of both a Lie algebra and a Lie coalgebra. These are compatible as a bialgebra on a certain natural subcomplex. In this paper I will generalize these two operations to the case of any cyclic operad, and show that they are each first in a series of higher order operations which fit together nicely and vanish on homology.

Let the graph complex corresponding to a cyclic operad $\mathcal{O}$ be denoted by $\mathcal{G}^{\mathcal{O}}$. I will define a sequence of "higher order brackets"

$$
\phi_{n}: S^{n} \mathcal{G}^{\mathcal{O}} \rightarrow \mathcal{G} \text {. }
$$


The map $\phi_{n}$ is defined by fusing together $n$ graphs along a $2 n$-gon in all possible ways (Figure 4). Extending each $\phi_{n}$ as a coderivation to $S \mathcal{G}^{\mathcal{O}}$, these maps are all compatible with each other in a very strong sense (Theorem 1 ). For any subset $I \subset \mathbb{N}$, define $\phi_{I}=\sum_{i \in I} \phi_{i}$. Theorem 1 implies that $\phi_{I}^{2}=0$. This is precisely the definition of a $\mathrm{Lie}_{\infty}$ (strong homotopy Lie) structure. In this way we get uncountably many $\mathrm{Lie}_{\infty}$ structures.

Let $P \mathcal{G}^{\mathcal{O}}$ denote the subcomplex of the graph complex spanned by connected graphs. I will define a sequence of "higher order cobrackets"

$$
\theta_{n}: P \mathcal{G}^{\mathcal{O}} \rightarrow S^{n} P \mathcal{G}^{\mathcal{O}} \text {. }
$$

The map $\theta_{n}$ is defined by fissioning a graph into $n$ graphs along a $2 n$-gon (Figure 5). The $\theta_{n}$ maps, extended to $S \mathcal{G}^{\mathcal{O}}$ as derivations, are compatible in a strong sense also (Theorem 2). For any $I \subset \mathbb{N}, \theta_{I}$ is defined as above, and Theorem 2 implies each $\phi_{I}$ is a co-Lie $\infty$ structure.

Trouble arises, as was foreshadowed in $[\mathbf{C V}]$ in the compatibility between brackets and cobrackets. In $[\mathbf{C V}]$ we were able to avoid difficulty by restricting to connected graphs without separating edges, and indeed in this context $\theta_{2}, \phi_{2}$ are compatible in a Lie bialgebra sense (Theorem 3). But there appears to be no similar way out for the higher order operations. The higher order brackets and cobrackets simply fit together in a more complicated way than one would guess, even on graphs without separating edges.

All of the operations are highly nontrivial on chains, and are compatible with the boundary operator. Indeed they vanish canonically on the level of homology. Thus these operations can be thought of as "generalized Schouten brackets," since in the case of Lie algebras, the Schouten bracket is an operation on the Chevalley-Eilenberg complex which vanishes canonically upon application of the homology functor.

Moira Chas and Dennis Sullivan [CS] define similar structures on string homology, the homology of a free loop space. They define an uncountable family of $\mathrm{Lie}_{\infty}$ structures, indexed by sets of positive integers, on string homology which obey the same compatibility relations as the ones found here (Theorem 1). They also find a Lie bialgebra structure $[\mathbf{C}]$ and [CS2]. Drawing the analogy further, one is led to speculate that string homology has an uncountable infinity of co-Lie $\infty$ structures. It would be interesting to know whether such co structures, if they exist, are compatible in a nice way with the Lie structures, or if they mirror more complicated graph interactions.

\section{Cyclic operads and graphs.}

We begin by briefly reviewing the salient features of a cyclic operad, and proceed to give Markl's construction of graph complexes. A good introduction to these objects can be found in the recent book by Markl, Shnider and Stasheff [MSS].

Kontsevich's three graph complexes are associated to the commutative, associative and Lie operads. Each of these operads $\mathcal{O}=\oplus \mathcal{O}(n)$ has a 
description as a vector space spanned by different flavors of rooted trees with labelled leaves.
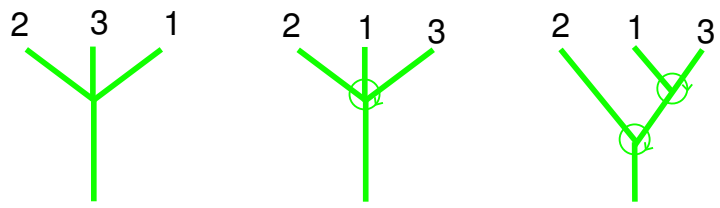

Figure 1. Elements of Comm[3], Assoc[3], and Lie[3] respectively.

- The $n$th degree part of the commutative operad $\operatorname{Comm}(n)$ has a basis consisting of rooted trees which have one internal vertex and $n$ labelled leaves. Hence Comm $[n]$ is 1 dimensional! The composition law $\operatorname{Comm}[m] \otimes \operatorname{Comm}\left[n_{1}\right] \otimes \cdots \otimes \operatorname{Comm}\left[n_{m}\right] \rightarrow \operatorname{Comm}\left[n_{1}+\cdots+n_{m}\right]$ is defined on $c \otimes c_{1} \otimes \cdots \otimes c_{m}$ by grafting the root of each $c_{i}$ onto the leaf of $c$ labelled by $i$ for each $i$, and suitably relabelling the leaves. The composition is completed by contracting all edges not adjacent to a root or a leaf.

- The $n$th degree part of the associative operad $\operatorname{Assoc}(n)$ has a basis consisting of rooted trees with one internal vertex which have a specified cyclic ordering of the edges incident to the vertex, and which have $n$ labelled leaves. Composition is again by grafting and contracting created edges, with the proviso that the cyclic ordering is respected.

- The $n$th degree part of the Lie operad $\operatorname{Lie}(n)$ is actually easiest to describe as a quotient space $\overline{\operatorname{Lie}(n)} / \mathrm{AS}+\mathrm{IHX} . \overline{\operatorname{Lie}(n)}$ has a basis given by rooted trivalent trees with $n$ labelled leaves, where each vertex has a specified cyclic order of adjacent edges. The AS subspace is spanned by sums $T_{1}+T_{2}$, where $T_{1,2}$ are identical except for a cyclic ordering on some vertex. Modding out by AS says that Lie algebras are anti-symmetric. The IHX subspace is spanned by sums $T_{1}-T_{2}+T_{3}$, where $T_{1,2,3}$ are identical trees except at one spot where they are as in Figure 2. On the level of Lie algebras this is the Jacobi relation. Composition is via grafting, but without the contraction step.

Notice that in each of these cases the action of the symmetric group $S_{n}$ which permutes the labels of the leaves can be extended to an action of $S_{n+1}$. This is by thinking of the root as another labelled leaf, say labelled by 0 . (One must check in the Lie case that the IHX subspace is preserved by this action.) Operads where this extension is possible are called cyclic [GK], provided that the extension satisfies appropriate axioms. Other examples of cyclic operads are the endomorphism operad and the Poisson operad.

As a general philosophy, one can think of cyclic operads as consisting of unrooted trees, with composition given by some version of grafting. The idea is to plug these in to the nodes of a graph to obtain different types of 

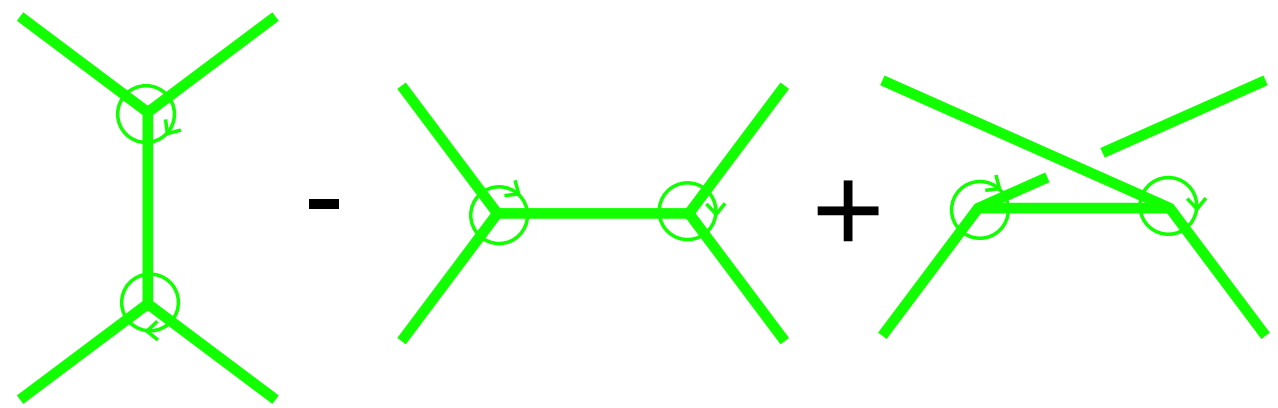

Figure 2. The IHX relation. Each term represents a piece of a graph which is identical outside of the pictured spot.

decorations on a graph. Plugging in a basis element from $\operatorname{Comm}(n)$ at each vertex of valence $n$, one simply gets an undecorated graph. Plugging in an element of $\operatorname{Assoc}(n)$ one gets a cyclic order at the vertex. This is often called a ribbon graph. Plugging in an element of $\operatorname{Lie}(n)$ gives a relatively strange object. By definition it is obtained from some unrooted ribbon trivalent trees by joining the leaves together with edges. See Figure 3. Thus one may think of it as a trivalent graph with a special distinguished subset where IHX and AS relations may take place. It is reminiscent of the diagram algebras that appear in the theory of Vassiliev invariants of low dimensional objects, which consist of (uni)trivalent graphs, but where the AS and IHX relations are not restricted to a distinguished subset.

In general, let $H(v)$ be the set of half-edges incident to a vertex. Let $L$ be a labelling of the elements of $H(v)$ by $0, \ldots, n$, where $n+1$ is the valence of $v$. Now define

$$
\mathcal{O}((H(v)))=\left(\oplus_{L} \mathcal{O}(n)\right)_{S_{n+1}}
$$

which is the set of coinvariants under the action of $S_{n+1}$, which acts as follows. If $o \in \mathcal{O}(n)$, let $o_{L}$ denote putting $o$ in the $L$ th summand of the direct sum. If $\sigma \in S_{n+1}$ then $\sigma \cdot o_{L}=(\sigma \cdot o)_{\sigma \cdot L}$. When $\mathcal{O}$ is an operad of trees, $\mathcal{O}((H(v)))$ is isomorphic to the space of identifications of the leaves and root of elements of $\mathcal{O}[n]$ with the half-edges incident to $v$.

Now we define an $\mathcal{O}$-labelling of a graph to be a choice of element $o_{v} \in$ $\mathcal{O}((H(v)))$ for each vertex, $v$, of the graph. Graphically, we put a circle at each vertex to represent the operad element.

In addition we would like a notion of "orientation" of a graph, which will make it possible to define a boundary operator. This is analogous to the need for an orientation of the simplices of a simplicial complex in order to do the same. There are many equivalent notions, perhaps the most intuitive is the following.

Definition. An orientation of a graph is an ordering of the vertices and a choice of direction for each edge, modulo the even action of $S_{V} \times \mathbb{Z}_{2}^{E}$. Here 
$V$ and $E$ are the number or vertices and edges of the graph, respectively. An element of $S_{V} \times \mathbb{Z}_{2}^{E}$ is called even if it is a product of an even number of elements each of which is either a transposition in $S_{v}$ or an element of the form $(0, \ldots, 1, \ldots, 0) \in \mathbb{Z}_{2}^{E}$.

Notice that any graph has exactly two orientations. Let - indicate the map switching orientations.

Remark. Lie graphs actually have a much simpler description, because the orientations of the graph and vertices cancel out to a large degree. Namely, one can think of a Lie graph as a trivalent graph with a distinguished subforest, whose edges are ordered modulo even permutations. The IHX relation in the Lie operad becomes the condition that the three terms in an IHX relation of the trivalent graph sum to zero provided the edge involved is in the forest. This will be explained carefully in [CV2].

2.1. Chain complexes. Now for any cyclic operad $\mathcal{O}$ we are ready to define $\mathcal{O}$-graph complexes.

Define $\mathcal{G}_{v}^{\mathcal{O}}$ to be the span of $\mathcal{O}$-labelled oriented graphs with vertices all of valence $\geq 3$, modulo the relation $(G$, or $)=-(G,-$ or $)$ and also modulo multilinearity of the $\mathcal{O}$-labels. More precisely, we set

$$
\mathcal{G}^{\mathcal{O}}=\left(\bigoplus_{(G, o r)} \bigotimes_{v \in V(G)} \mathcal{O}((H(v)))\right) /\{(G, o r)=-(G,-o r)\}
$$

where the direct sum is over oriented graphs with vertices of valence $\geq 3$, and where $V(G)$ is the set of vertices of $G$. Define $\mathcal{G}_{v}^{\mathcal{O}}$ to be the part of $\mathcal{G}^{\mathcal{O}}$ with $v$ vertices.

For each edge, $e$, in a graph $(G, o r)$ we define contraction along that edge $(G, \text { or })_{e}$ to be the graph where the two operad elements at each endpoint of $e$ are composed along $e$. The induced orientation can be fixed by assuming that the endpoints of $e$ are labelled 1 and 2 and the edge direction is from 1 to 2. The new vertex, which results from composing the two operad elements, is labelled 1, and all other indices are reduced by 1 . If $e$ is a loop, then define $(G, \text { or })_{e}=0$. In the commutative case, $(G, \text { or })_{e}$ is defined by simply contracting the edge of the (undecorated) graph. In the associative case the cyclic orders at both endpoints of an edge are joined together to give a cyclic order at the vertex resulting from the edge collapse. For an example in the Lie case, see Figure 3.

Define $\partial_{G}: \mathcal{G}_{v}^{\mathcal{O}} \rightarrow \mathcal{G}_{v-1}^{\mathcal{O}}$ by $\partial_{G}(G$, or $)=\sum_{e \in E(G)}(G, \text { or })_{e}$, where $E(G)$ is the set of edges of $G$.

Remark. In the simpler version of the Lie case, the boundary operator adds an edge to the forest in all possible ways, with the edge's number coming directly after the edge numbers in the original forest. 

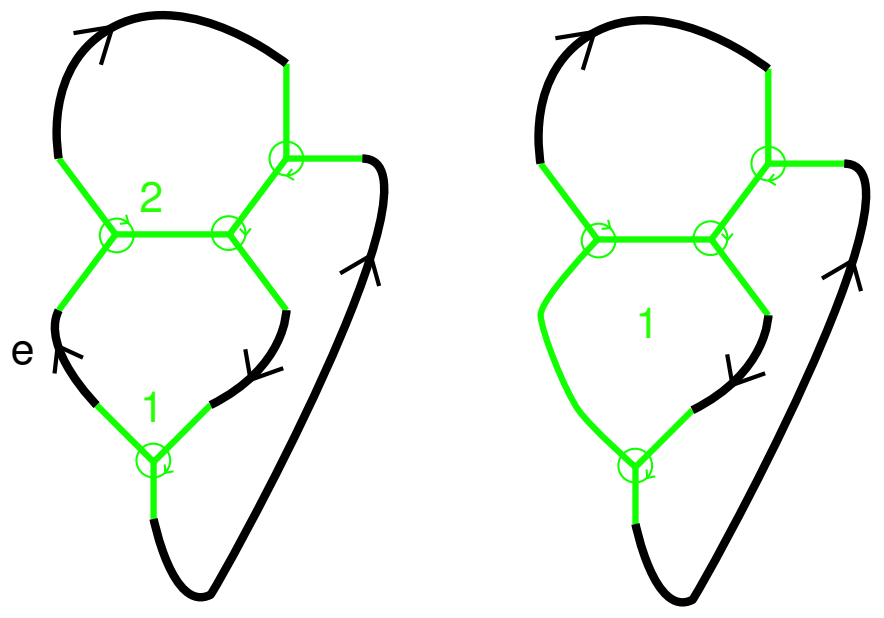

Figure 3. The Lie graphs $G$ and $G_{e}$.

$\mathcal{G}^{\mathcal{O}}$ is a graded commutative algebra under disjoint union. It is also a graded commutative coalgebra, defining the coproduct such that connected graphs are primitive, and extending multiplicatively. Thus we may write $P \mathcal{G}^{\mathcal{O}}$ for the subspace generated by connected graphs. Let $P^{(n)} \mathcal{G}^{\mathcal{O}}$ be the subspace generated by connected graphs with $b_{1}=n$.

Even though this paper is concerned with chain complexes and not their homology per se, it is still useful to record the following facts.

Let $\operatorname{Out}\left(F_{n}\right)$ denote the group of outer automorphisms of the free group $F_{n}$, and let $\mathcal{M}_{g, m}^{\prime}$ denote the moduli space of a surface of genus $g$ with $m$ unlabelled punctures.

Then

$$
\begin{aligned}
H_{k}\left(P^{(n)} \mathcal{G}^{\mathcal{A} s s o c}\right) & =\bigoplus_{m \geq 1, g: 2 g+m-1=n} H^{4 g-4+2 m-k}\left(\mathcal{M}_{g, m}^{\prime} ; \mathbb{Q}\right) \\
H_{k}\left(P^{(n)} \mathcal{G}^{\mathcal{L} i e}\right) & =H^{2 n-2-k}\left(\operatorname{Out}\left(F_{n}\right) ; \mathbb{Q}\right) .
\end{aligned}
$$

In addition, part of commutative graph cohomology plays a role in the theory of finite type invariants of homology 3 -spheres. More precisely, we have that

$$
\bigoplus_{n \geq 2} H^{2 n-2}\left(P^{(n)} \mathcal{G}^{\mathrm{Comm}}\right) \cong P \mathcal{A}(\emptyset)
$$

where $P \mathcal{A}(\emptyset)$ is the diagram algebra where the logarithm of the Aarhus version of the LMO invariant takes values [B-NGRT].

The first two statements above are due to Kontsevich, being implicit in his paper $[\mathbf{K}]$. A more detailed explanation of these two facts and their 
proofs will appear in [CV2]. The last statement, the relation to finite type invariants, is essentially content-free, being a trivial isomorphism, at least modulo equivalences of various notions of orientation.

2.2. Cohomology. In at least two interesting cases, it is possible to define graph cohomology. The coboundary operator $\delta$ is the sum of inserting an edge in all possible ways. In the commutative and associative cases this makes perfect sense. Unfortunately, in the Lie case an insertion, which is essentially the deletion of an edge from the forest, does not preserve the IHX subspace and is not well-defined. In the cases where $\delta$ can be defined the boundary and coboundary are adjoint with respect to the inner product $\langle G, H\rangle=|\operatorname{Aut}(G)| \delta_{G H}$. This can be seen by applying the argument of [CV], Proposition 12 mutatis mutandis.

\section{Fusion.}

We start with an oriented labelled $2 n$-gon. Label every other edge on its perimeter consecutively by the numbers $1 \ldots n$, consistent with the orientation. Now fix $n$ directed edges $e_{1}, \ldots, e_{n}$ of a graph $G$. Define $G\left\langle e_{1}, \ldots, e_{n}\right\rangle$ to be the graph formed in the following way. First, for each $i$, glue the edge marked $i$ of the $2 n$-gon to the edge $e_{i}$ of the graph. Second, delete these edges along which the $2 n$-gon was attached leaving $n$ new edges. This is illustrated in Figure 4. The graph $G\left\langle e_{1}, \ldots, e_{n}\right\rangle$ has an induced orientation which can be easily described. Fix a labelling of the graph such that the directions of the edges $e_{1}, \ldots, e_{n}$ are both consistent with the graph's orientation and with the directions which correspond to the gluing. The $n$ new edges have orientations induced by the $n$-gon. Switch all of these, as is usual with a cobordism.
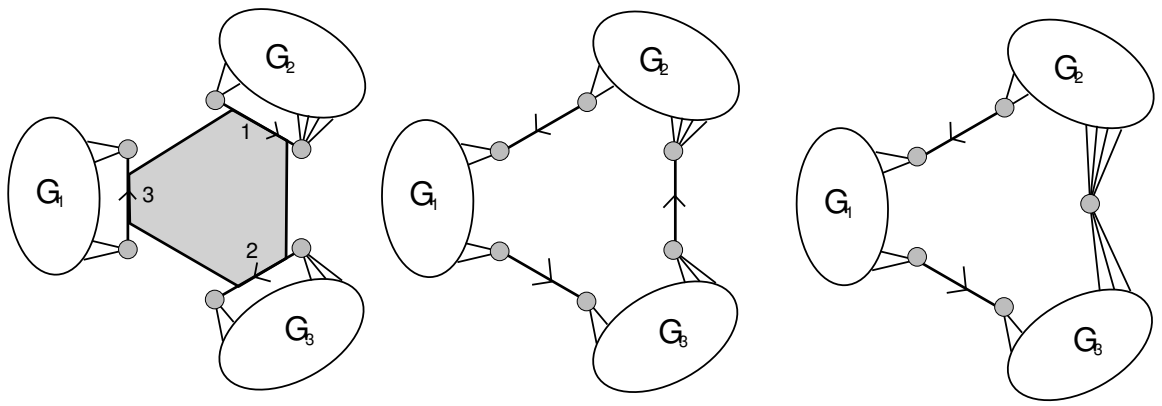

Figure 4. One term in $\phi_{3}\left(G_{1}, G_{2}, G_{3}\right)$.

Now, for any $n \in \mathbb{N}$ we define an operation

$$
\phi_{n}: S^{n} \mathcal{G}^{\mathcal{O}} \rightarrow \mathcal{G}^{\mathcal{O}}
$$

by $\phi_{n}\left(G_{1} \odot \cdots \odot G_{n}\right)=\sum\left(G_{1} \cdot G_{2} \cdots G_{n}\right)\left\langle e_{1}, \ldots, e_{n}\right\rangle_{e}$, where: 
- The sum is over all $n$-tuples of directed edges $\left(e_{1}, e_{2}, \ldots, e_{n}\right)$ all of which Lie in separate $G_{i}$.

- The notation "๑" denotes "graded symmetric tensor product."

- The edge $e$ which is contracted is the edge coming from the boundary of the $2 n$-gon between " 1 " and " $2 . "$

Thus $\phi_{n}$ is a type of fusion operation which takes $n$ graphs and fuses them together along a $2 n$-gon.

Extend $\phi_{n}$ to $S \mathcal{G}^{\mathcal{O}}$ as a coderivation. That is

$$
\phi_{n}\left(G_{1} \odot \cdots \odot G_{k}\right)=\sum_{I \cup J} \epsilon(I, J) \phi_{n}\left(G_{I}\right) \odot G_{J},
$$

where $I, J$ is an ordered partition of $1, \ldots, k$, with $|I|=n$, and $\epsilon(I, J)$ is the sign defined by the equation $G_{1} \odot \cdots \odot G_{k}=\epsilon(I, J) G_{I} \odot G_{J}$. Notice that $\phi_{1}$ by definition glues on a bigon to an edge, which doesn't change the edge, and then contracts it. That is, $\phi_{1}=\partial_{G}$. Notice that it doesn't matter whether we extend $\partial_{G}$ to $S \mathcal{G}^{\mathcal{O}}$ as a derivation or a coderivation, since they are equivalent in this case!

Theorem 1. The following equations hold:

a) $\forall i \phi_{i}^{2}=0$,

b) $\forall i \neq j \phi_{i} \phi_{j}+\phi_{j} \phi_{i}=0$.

Corollary 1. Let $I$ be a subset of $\mathbb{N}$, finite or infinite. Let $\phi_{I}=\sum_{i \in I} \phi_{i}$. Then $\phi_{I}^{2}=0$.

Proof of Theorem 1. First we show $\left.\phi_{n}^{2}\right|_{S^{k} \mathcal{G} \mathcal{O}}=0$. We only need consider the case when $k=2 n-1$, which implies the higher degree cases.

$$
\phi_{n}^{2}\left(G_{1} \odot \cdots \odot G_{2 n-1}\right)=\sum_{I \cup J=[2 n-1]} \phi_{n}\left(\phi_{n}\left(G_{I}\right) \odot G_{J}\right) .
$$

Thus we are attaching a disk to $G_{i}$ where $i \in I$ along its $n$ subarcs. We then attach a disk to the result together with the other $n-1$ graphs. If the second disk attaches to an edge not involved in the first disk, then this gives the same unoriented result as attaching the disks in the other order. Keeping track of the orientation, we see that the two orders of attaching the disk cancel. The other possibility is that the second disk attaches to the first. This can be thought of as attaching a $4 n-2$-gon to the $2 n-1$ graphs, with a separating arc along the $4 n-2$-gon, and two ordered edges marked for collapse. We can simplify the combinatorics somewhat by shrinking the complement of the $2 n-1$ attaching regions for the disk, to get a $2 n-1$ gon with an arc joining two vertices and two vertices marked for collapse. The sorts of configurations that arise are exactly recorded by the concept of admissible defined below. The lemma now follows from the following analysis of $2 n-1$-gons. 
Define $\operatorname{Conf}(2 n-1, n)$ be the set of admissible configurations of a $2 n-1$ gon. An admissible configuration consists of an embedded arc on the $2 n-1$ gon between two of the vertices, thereby partitioning the $2 n-1$ vertices into two sets of $n-1$ and $n-2$ respectively, on each side of the arc. There are also two vertices labelled by 1 and 2 , the 1 must be in the set of $n-1$ and the 2 must be among the $n-2$ or it could be one of the endpoints of the arc. We claim that the subset of $\operatorname{Conf}(2 n-1, n)$ where two specific vertices are marked 1 and 2 is bijective with the subset where these vertices are marked 2 and 1, respectively. This follows from the fact that there is a unique automorphism exchanging any two vertices of a $2 n-1$-gon. This induces a bijection between the two types of configurations. Keeping track of orientations, we see that the terms of corresponding to elements of $\operatorname{Conf}(2 n-1, n)$ cancel in pairs.

The fact that $\phi_{i}, \phi_{j}$ anti-commute follows from the following similar facts about configurations of $i+j-1$-gons, $\operatorname{Conf}(i+j-1, i, j)$. The arc in this case will partition the vertices into a set of $i-1$, and a set of $j-2$, where the 1 vertex must Lie in the $i-1$ and the 2 elsewhere. We claim there is a bijective correspondence between subsets of $\operatorname{Conf}(i+j-1, i, j)$ where two fixed vertices are labelled 1 and 2 and the subsets of $\operatorname{Conf}(i+j-1, j, i)$ where these vertices are labelled 2 and 1. To see this, fix an automorphism of the $i+j-1$-gon, exchanging the two given vertices. This will carry one set of configurations onto the other.

Proposition 1. $\phi_{n}$ is canonically zero at the level of homology.

Proof. The fact that $\phi_{n}$ is even compatible with homology is the fact

$$
\partial_{G} \circ \phi_{n}+\phi_{n} \circ \partial_{G}=0
$$

where $\partial_{G}$ is extended to $S \mathcal{G}^{\mathcal{O}}$ as a derivation. This follows since $\partial_{G}=\phi_{1}$.

It remains to show that it vanishes canonically. Consider the map

$$
\mu_{n}: S^{n} \mathcal{G}^{\mathcal{O}} \rightarrow \mathcal{G}^{\mathcal{O}}
$$

which is defined by gluing in a $2 n$-gon in all possible ways, but without contracting an edge. Then a straightforward argument shows that $\phi_{n}=$ $\partial_{G} \mu_{n}-\mu_{n} \partial_{G}$. Thus if the input to $\phi_{n}$ consists of $n$ cycles, the $\mu_{n} \partial_{G}$ term in this equation vanishes, and what is left expresses $\phi_{n}$ as a boundary.

\section{Fission.}

In this section, for simplicity, we restrict ourselves to connected graphs, although much of it can be generalized to the nonconnected case. In particular, when edge insertions make sense, one can dualize and prove Theorem 2 analogously to Proposition 11 of $[\mathbf{C V}]$.

Note that $\mathcal{G}^{\mathcal{O}} \cong S\left(P \mathcal{G}^{\mathcal{O}}\right)$. Denote this isomorphism by $S$. Let

$$
\pi_{i}: S\left(P \mathcal{G}^{\mathcal{O}}\right) \rightarrow S^{i}\left(P \mathcal{G}^{\mathcal{O}}\right)
$$


be the natural projection. Define the map

$$
\partial_{i}: \mathcal{G}_{v}^{\mathcal{O}} \rightarrow \mathcal{G}_{v-1}^{\mathcal{O}}
$$

by summing over all ways of attaching a $2 i$-gon to the edges of an $\mathcal{O}$-graph, and then contracting the edge between 1 and 2 . The behavior of this operator (which does not have square zero) is complicated, but it becomes better behaved if we look at the part which disconnects the graph the most.

Definition. The map

$$
\theta_{i}: P \mathcal{G}^{\mathcal{O}} \rightarrow S^{i}\left(P \mathcal{G}^{\mathcal{O}}\right)
$$

is defined as the composition $\frac{1}{2} \pi_{i} \circ S \circ \partial_{i}$.

The operator $\theta_{i}$ can be thought of as a type of fission, where a graph splits up into $i$ particles. See Figure 5.
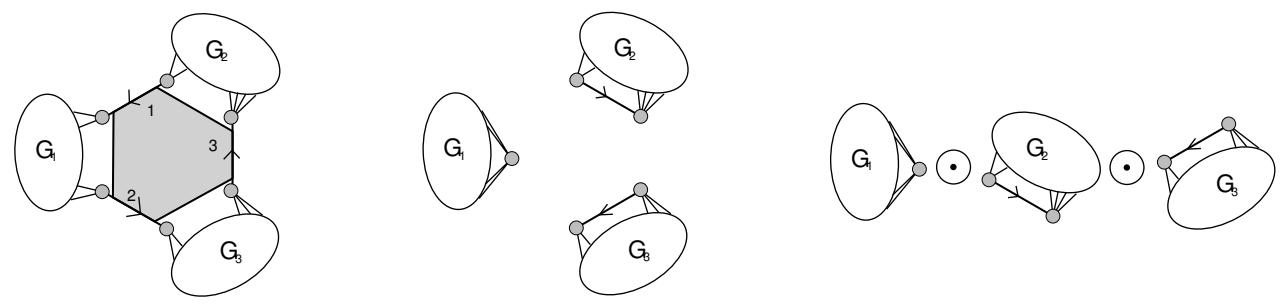

Figure 5. A term in $\theta_{3}(G)$. The middle picture represents a term in $\partial_{3}(G)$, and the final picture is a result of applying $S$.

Extend $\theta_{i}$ to

$$
\theta_{i}: S\left(P \mathcal{G}^{\mathcal{O}}\right) \rightarrow S\left(P \mathcal{G}^{\mathcal{O}}\right)
$$

as a derivation. Notice that $\theta_{1}=\partial_{G}=\phi_{1}$.

Theorem 2. The following identities hold:

a) $\forall i \neq j \theta_{i} \theta_{j}+\theta_{j} \theta_{j}=0$,

b) $\forall i \theta_{i}^{2}=0$.

Proof. We prove a). Statement b) is similar. We show that

$$
\theta_{i} \theta_{j}+\theta_{j} \theta_{i}: \mathcal{G}^{\mathcal{O}} \rightarrow S^{i+j}\left(\mathcal{G}^{\mathcal{O}}\right)
$$

is zero, which is enough. If the $i$-gon and $j$-gon attach to two different sets of edges, they can be applied in either order to get the same (unoriented) result. Keeping track of orientation, one sees that they anticommute. Attaching one disk, and then the other to an edge of the original disk is the same as adding a bigger disk with an ordered pair of two sides marked for collapse. We may now apply our analysis from the proof of Theorem 1 to show that the terms cancel in pairs. 
Corollary 2. Let I be a subset of $\mathbb{N}$, finite or infinite. Let $\theta_{I}=\sum_{i \in I} \theta_{i}$. Then $\theta_{I}^{2}=0$.

Proposition 2. $\theta_{i}$ is canonically zero at the level of homology.

Proof. That $\theta_{i}$ is compatible with homology follows since $\theta_{1}=\partial_{G}$.

A similar argument to Proposition 1 shows that $\theta_{i}$ vanishes canonically on homology.

The operator $\theta_{i}$ can be defined for disconnected graphs as well, as we alluded to earlier. Suppose we start with a graph with $k$ connected components. A $2 i$-gon attaches to one of these and it fissions into $i$ components. In order to get a well-defined map, the remaining $k-1$ components must be distributed with the $i$ fission components in all possible ways, which leads to more complicated formulas.

\section{Compatibility.}

It is unclear if there is a theory of Lie $_{\infty}$ bialgebras; a search of MathSciNet yields no hits. Under some obvious generalizations of the definition of Lie bialgebra to the case of higher order operations on the symmetric algebra, the higher degree fusion operations are not compatible with the higher degree fission operations. Interestingly, degree 2 fission is compatible with degree 2 fusion on the subcomplex of connected graphs with no separating edges. As was noted in $[\mathbf{C V}]$ this is not the case on the full complex $\mathcal{G}^{\mathcal{O}}$.

Definition. Let $P^{\text {irred }} \mathcal{G}^{\mathcal{O}}$ be the subcomplex of $\mathcal{G}^{\mathcal{O}}$ spanned by connected (primitive) graphs with no separating edges (irreducible).

Theorem 3. On $P^{\text {irred }} \mathcal{G}^{\mathcal{O}}$ the following equation holds:

$$
\theta_{2} \phi_{2}(X \odot Y)+\phi_{2}\left(\theta_{2}(X) \odot Y\right)+(-1)^{x} \phi_{2}\left(X \odot \theta_{2}(Y)\right)=0 .
$$

Proof. The bracket $\phi_{2}$ and cobracket $\theta_{2}$ coincide with the operations $[\cdot, \cdot]$ and $\theta$ defined in $[\mathbf{C V}]$ for the commutative operad. In that paper, we defined everything in terms of contracting pairs of half-edges, but the operations are easily seen to match. (In fact, we mentioned a "dotted line notation" in that paper which is very close to the definition of $\phi_{2}$ considered here.) Now use the argument from [CV] Theorem 1, which holds even if the vertices are labelled by the operad $\mathcal{O}$.

Acknowledgements. It is a pleasure to thank Karen Vogtmann for many discussions. I also wish to thank Swapneel Mahajan for his perceptive input. Credit also goes to the anonymous referee who noticed an error in the original manuscript and suggested many expositional improvements. 


\section{References}

[B-NGRT] D. Bar-Natan, S. Garoufalidis, L. Rozansky and D.P. Thurston, The Aarhus integral of rational homology 3-spheres I: A highly nontrivial flat connection on $S^{3}$, to appear in Selecta Mathematica, see also q-alg/9706004.

[C] M. Chas, Combinatorial Lie bialgebras of curves on surfaces. Preprint 2001, math.GT/0105178.

[CS] M. Chas and D. Sullivan, String topology. Preprint 1999, math.GT/9911159.

[CS2] L Lie bialgebras of closed strings in manifolds. Preprint.

[CV] J. Conant and K. Vogtmann, Infinitesimal operations on graph complexes. Preprint, math.QA/0111198.

[CV2] , in preparation.

$[\mathrm{CuV}] \quad$ M. Culler and K. Vogtmann, Moduli of graphs and automorphisms of free groups, Invent. Math., 84(1) (1986), 91-119, MR 87f:20048, Zbl 0589.20022.

[GK] E. Getzler and M. Kapranov, Cyclic operads and cyclic homology, Geometry, Topology, and Physics, 167-201, Conf. Proc. Lecture Notes Geom. Topology, IV, Internat. Press, Cambridge, MA, 1995, MR 96m:19011, Zbl 0883.18013.

[GK2] _ Modular operads, Compositio Math., 110(1) (1998), 65-126, MR 99f:18009, Zbl 0894.18005.

[K] M. Kontsevich, Formal (non) commutative symplectic geometry, The Gelfand Mathematical Seminars, 1990-1992, 173-187, Birkhäuser Boston, Boston, MA, 1993, MR 94i:58212, Zbl 0821.58018.

[M] M. Markl, Cyclic operads and homology of graph complexes, Rendiconti del Circolo Matematico di Palermo Serie II, Suppl., 59 (1999), 161-170, MR 2000g:18009, Zbl 0970.18011.

[MSS] M. Markl, S. Shnider and J. Stasheff, Operads in Algebra, Topology and Physics, Mathematical Surveys and Monographs, 96, American Mathematical Society, 2002, CMP 1898414.

[P] R.C. Penner, Perturbative series and the moduli space of Riemann surfaces, J. Differential Geom., 27(1) (1988), 35-53, MR 89h:32045, Zbl 0608.30046.

[V] A. Voronov, Notes on universal algebra. Preprint, math.QA/0111009.

Received April 4, 2002 and revised July 25, 2002. This work was partially supported by NSF VIGRE grant DMS-9983660.

Department of Mathematics

Cornell University

ITHACA, NY 14853-4201

E-mail address: jconant@math.cornell.edu 